\title{
ROBUSTNESS OF STEEL AND COMPOSITE BUILDINGS SUFFERING THE DYNAMIC LOSS OF A COLUMN
}

\begin{abstract}
In case a vehicle impacts a building frame, one or several columns may be damaged or even completely destroyed. Nowadays, it is a concern to mitigate the risk of progressive collapse of the whole structure further to such a localised exceptional event. Although this robustness requirement is part of several design codes, very few precise practical guidelines are provided, especially as far as dynamic failures are concerned. The present research focuses on building frames suffering the loss of one internal column. The dynamic response is more specifically investigated, with the aim to understand this behaviour in order to eventually derive simplified procedures for robustness assessment.

This paper first briefly presents the main previous achievements related to the prediction of the static response of a plane frame suffering a column loss. The investigations into the dynamic behaviour are then summarised, which constitutes the core topic of the paper. For sake of simplicity, the dynamic response is described using a basic substructure that was proved to show many similarities in behaviour with a global frame losing a column. A simplified model is finally developed for the prediction of the considered system dynamic response.
\end{abstract}

Keywords: Robustness, Building frames, Column loss, Impact, Dynamic effects.

\section{INTRODUCTION}

Generally speaking, three main reference approaches exist to face the structural robustness issue: the indirect "tying" method which provides prescriptive rules, and two direct methods ("specific local resistance method" and "alternative load path method") which are based on particular scenarios and require a structural analysis. In the present study, the so-called "alternative load path method" is followed and the conventional scenario "loss of a column" is considered. Basically, it means that a column is theoretically removed from the structure and the redistribution of forces within the frame is investigated to determine whether a final stable state can be reached.

The loss of a column in a building frame can be caused by different types of exceptional events: explosion, impact of a vehicle, fire... Amongst these actions, some induce a sufficiently low decrease of the column bearing capacity for a static response of the frame to be assumed. For example, it is the case when a localised fire leads to a highly progressive diminution of the mechanical properties of a column. However, in many other circumstances such as impacts, the column is relatively quickly removed inducing significant dynamic effects that must be taken into account.

Different design codes include robustness requirements (Refs. [1], [2], [3], [4] and [5]). However, there is an obvious lack of simple, though accurate, procedures to assess building robustness for practical use, even considering static behaviour of the structure. As far as the 
dynamic effects are concerned, still fewer indications are given. And when it comes to simplified methods considering the dynamic amplifications, only very rough rules are suggested such as performing a static analysis under the applied loads multiplied by two (Ref. [2] and [3]). This last rule gives correct results only in the very restrictive case of a perfectly elastic response under instantaneous loading. Such conditions are practically never fulfilled and applying this simplistic procedure leads to results which might be far too conservative in some cases but also, which is worse, unsafe under some other circumstances.

In 2005, Ellingwood and Dusenberry provided a literature review in the field of robustness summarising the information available in some reference papers as well as in national codes and standards from North America and Europe [6]. At the end of this paper, research issues and challenges are identified, amongst which the development of practical tools for design engineers. Indeed, although the structural response of a frame losing a column can be studied using finite element analyses, this approach is not convenient for practical use: such analyses have to account for both second order effects and plastic behaviour, which makes them quite difficult to perform, especially when dynamic effects have to be included. That is why practical simplified procedures need to be developed.

The research that has been conducted at the University of Liège for the last decade clearly aims at achieving this goal. In two recent $\mathrm{PhD}$ theses ([7] and [8]), a complete analytical procedure permitting the prediction of the static response of steel or composite plane frames further to a column removal was developed. More recently, complementary research has been undertaken with the objective to address the dynamic effects ([9], [10], [11]).

In this paper, the global approach followed to study the response of a building frame losing a column is first presented, as well as previous research into the static behaviour of such structures further to this event. In particular, a substructure able to reproduce the response of a 2D frame after the formation of the global beam plastic mechanism induced by a column loss is defined. Then, the dynamic behaviour of such a substructure is investigated in details and the influence of different parameters (such as the first vibration period and the loading conditions) is highlighted. Finally, a simplified approach is developed for the prediction of the system maximum displacement and subsequently the internal forces developing in the substructure, permitting the definition of ductility and resistance requirements for robustness.

\section{GLOBAL APPROACH AND PREVIOUS RESEARCH}

A structure suffering the loss of a column can be decomposed in two parts: (i) the directly affected part, which is composed of the beams (together with the joints at their extremities) and the columns above the lost column; and (ii) the indirectly affected part, which consists of the rest of the frame (Fig. 1).

The static evolution of the vertical displacement $\Delta_{A}$ at the top of the failing column versus the axial load $N_{l o}$ that it supports is represented by a curve as illustrated in Fig. 2 . The behaviour can be divided in three main phases. The first one corresponds to the application of the service loads on the structure. It goes from point (1) (unloaded, $\Delta_{A}=0$ ) to point (2) (conventional loading, $\Delta_{A}<<$ ). The structure is assumed to remain elastic during this phase. At point (2), the exceptional event occurs and thus the column "AB" is progressively 
removed. In practice, the compression that the column sustains starts to decrease. During phase 2, the directly affected part goes from a fully elastic behaviour to a plastic mechanism. The first plastic hinge appears at point (3) while the complete plastic mechanism is formed at point (4). After this point, $\Delta_{A}$ rapidly increases and the second order effects become important. In particular, significant catenary actions develop in the bottom beams of the directly affected part implying the increase of the system stiffness and the decrease of the deformation rate (due to the activation of the beams axial stiffness). Eventually, if the frame exhibits sufficient robustness, and this is the topic of this study, a final stable state is obtained at point (5), when $N_{l o}=0$.

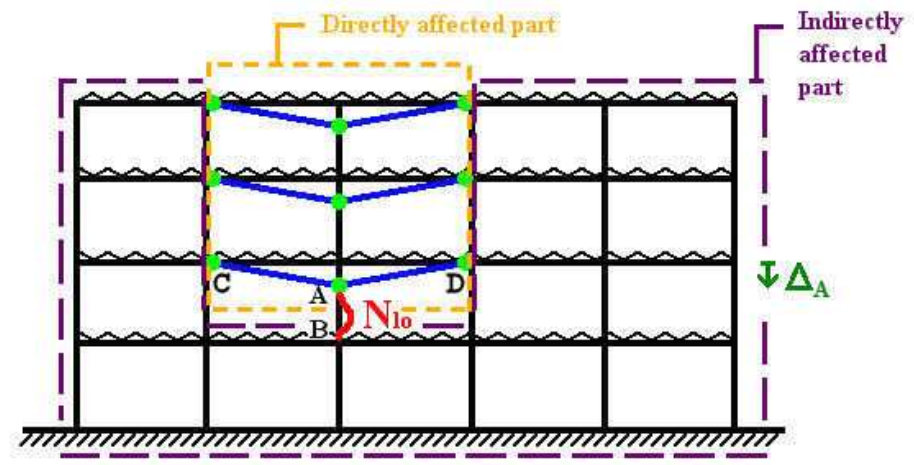

Fig. 1: Directly and indirectly affected parts of a building frame / Plastic mechanism caused by the loss of a column

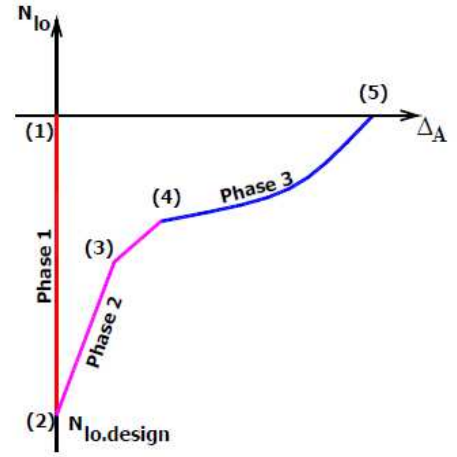

Fig. 2: Evolution of the vertical displacement $\Delta_{A}$ versus the axial load $N_{l o}$

It is only possible to reach a final stable state if no premature failure occurs, be it in the directly affected part or in the rest of the structure. It is thus necessary that:

the loads transferred from the directly affected part to the indirectly affected part do not induce the collapse of structural elements in the latter (buckling of columns or global plastic mechanism for instance),

- the compression supported by the upper beams of the directly affected part due to an "arch effect" does not lead to their buckling,

- the different structural elements of the directly affected part exhibit sufficient resistance and ductility to reach the displacement $\Delta_{A,(5)}$.

The static response of the structure during phases 1 and 2 as well as the behaviour of the indirectly affected part during phase 3 were investigated in [8] while the response of the directly affected part during phase 3 was studied in [7]. During this last phase, the global plastic mechanism is formed in the directly affected part and large displacements are observed. As a consequence, the second order effects become significant. In particular, tension loads develop in the bottom beams of the directly affected part. It was shown that the membrane forces developing in the two beams overhanging directly the lost column ("CA" and "AD" in Fig. 1) are significantly more important than the ones developing in the other beams. That is why the behaviour of this single storey was specifically investigated. For that purpose, a simplified substructure consisting of this double-beam and its beam-to-column joints is extracted from the frame. This substructure is represented in Fig. 3, where $Q$ is the downward force simulating the column loss. The influence of the indirectly affected part is modelled using an elastic horizontal spring with an adequately chosen stiffness $K$ and resistance $F_{R d}$. Analytical methods aiming at estimating the value of these parameters were developed; and the ability of such a substructure to reproduce the behaviour of the directly 
affected part with sufficient accuracy was validated against numerical tests. Finally, on the basis of the substructure, an analytical approach was developed to predict the response of the frame during phase 3 . Consequently, the static curve $\left(\Delta_{A}, N_{l o}\right)$ can be entirely derived using the combination of the developments detailed in [7] and [8], which provides a global analytical procedure. However, it is only valid as long as a static response of the frame is observed.

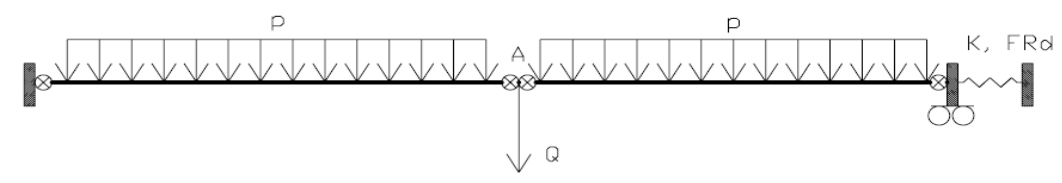

Fig. 3: Double-beam simplified substructure

\section{DYNAMIC BEHAVIOUR OF A SUBSTRUCTURE}

\subsection{Description of the considered substructure and loading conditions}

The dynamic behaviour of a simple substructure such as previously described was investigated using numerical simulations performed with the homemade finite element software Finelg (Ref. [12]), under the following hypotheses:

- steel structures are considered,

- the material behaviour law is admitted to be independent of the strain rate and a quasi-static elastic-perfectly plastic law is assumed (infinite ductility),

- the stiffness $K$ of the lateral spring remains constant and a high value of its resistance $F_{R d}$ is assumed,

. the beam-to-column joints are perfectly rigid and fully resistant.

A uniformly distributed load $p$ is applied on the double-beam. Initially, the central support is present and sustains a force $N_{0}\left(N_{0}=p \cdot l_{0}, l_{0}\right.$ being the initial length of each beam). Then, this support is progressively removed, which is modelled by applying a force $P$ equal and opposite to $N_{0}$ at mid length of the double-beam (Fig. 4(a)). The complete loss of the support lasts a time $t_{r}$ and a linear decrease of the force it sustains is assumed (Fig. 5). Under static conditions, it was shown in [7] that the uniformly distributed load $p$ can be neglected as far as the behaviour in phase 3 is investigated, i.e. for $P$ greater than $P_{p l}$, which is the force corresponding to the plastic plateau in the static curve (development of a beam mechanism). Indeed, for such values of the force, the static displacement is the same whether the distributed load $p$ is considered or whether it is taken equal to zero and the concentrated load $P$ is applied alone. The validity of this assertion for dynamic situations was studied in [9]. For that purpose, numerical dynamic models of a substructure were completed in order to compare the maximum displacement obtained in the two loading situations (Fig. 4) considering the same loading parameters $P$ and $t_{r}$. It was observed that the difference is limited provided that the force $P$ is large enough (above the static plastic plateau). That is the reason why the behaviour of the substructure under the simplified loading situation is investigated herein. Moreover, it was also shown in [9] that the introduction of damping in the system, though influencing its general response, does not induce a significant decrease of the maximum displacement. As a consequence, undamped systems are considered, which constitutes a conservative approach. 


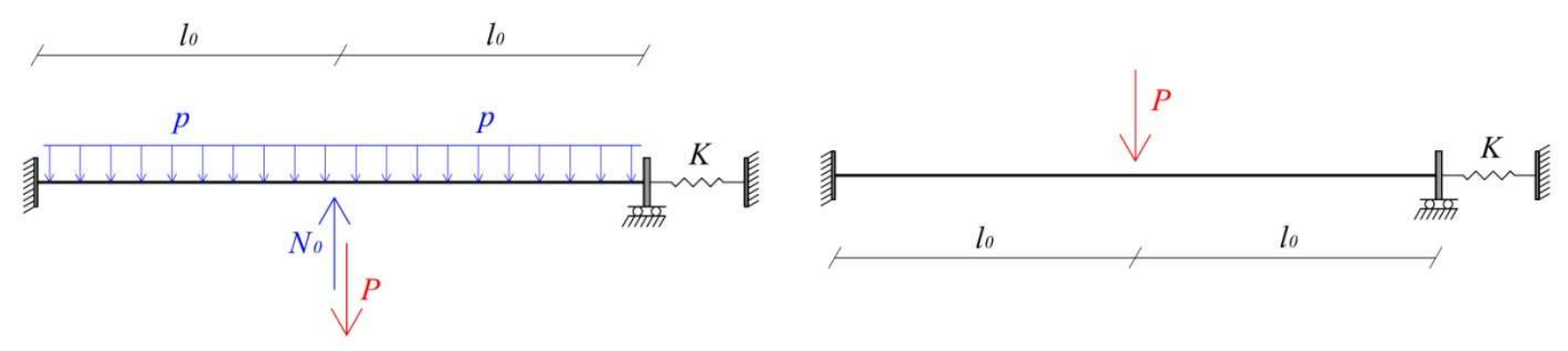

Fig. 4: Considered system with realistic (a) and simplified (b) loadings

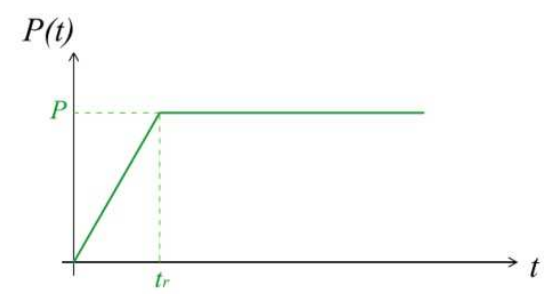

Fig. 5: Time evolution of the applied force $P(t)$

\subsection{Influence of different parameters on the dynamic behaviour}

To investigate the dynamic response of the substructure, a simplified loading consisting of a single concentrated load applied at mid length of the system is considered (Fig. 4(b)). Obviously, as well as in the static case, the level of the load $P$ and the geometrical and mechanical characteristics of the structure have an influence on its behaviour. In case of dynamic loadings, the application rate of $P$, characterised by the rise time $t_{r}$ (Fig. 5), is also of great importance. Besides, mass and damping properties are essential factors on which the dynamic response depends.

As mentioned above, the studied systems are undamped ones. As far as the mass influence is concerned, a change in mass will first affect the principal natural period of the system. In fact, numerical tests performed in the frame of the present research proved that the dynamic response of a given structure is governed by two parameters: $P$ and $t_{r} / T$, where $T$ is the period of the principal eigenmode in the elastic domain. Thus, if the mass of the system is modified but the rise time of the load is adapted so that the $t_{r}$ to $T$ ratio is kept constant, then the maximum displacement remains unchanged. Furthermore, the time evolution of the displacement remains the same provided that it is expressed as a function of a nondimensional time $t / T$ ( or $t / t_{r}$ ).

In [9], the behaviour of the substructure according to the loading parameters $P$ and $t_{r}$ (or $\left.t_{r} / T\right)$ was investigated through numerical dynamic analyses. All the results presented below are related to the following particular substructure:

$$
\begin{aligned}
& \text { beams: } l_{0}=6,5 \mathrm{~m}, \mathrm{IPE} 450, \mathrm{~S} 235, \mathrm{~m}=3000 \mathrm{~kg} / \mathrm{m}(T=0,31 \mathrm{sec}) \text {; } \\
& \text { - spring: stiffness } K=10000 \mathrm{kN} / \mathrm{m} \text {, resistance } F_{R d}>>\text {. }
\end{aligned}
$$

The value of the static force causing the formation of the beam plastic mechanism in this substructure is $P_{p l}=248 \mathrm{kN}$, as it can be observed in the static curve given in Fig. 6 .

Several dynamic analyses for different loading conditions $\left(P, t_{r}\right)$ were achieved, and the maximum displacement $u_{\max }$ recorded for each of them. It was thus possible to establish curves providing $u_{\max }$ as a function of the applied force $P$ for different values of $t_{r}$ (constant 
along one curve), see Fig. 6. In these curves, only dynamic loadings leading to $u_{\max }$ smaller than the displacement corresponding to the complete yielding of the beams in tension are considered. The upper yellow curve corresponds to the static case, while the lower blue curve is the so-called pseudo-static one, which provides the maximum displacement reached if $P$ is applied instantaneously $\left(t_{r}=0\right)$. Such a curve can easily be established using the nonlinear static curve, following a procedure developed at the Imperial College London (Refs. [13] and [14]).

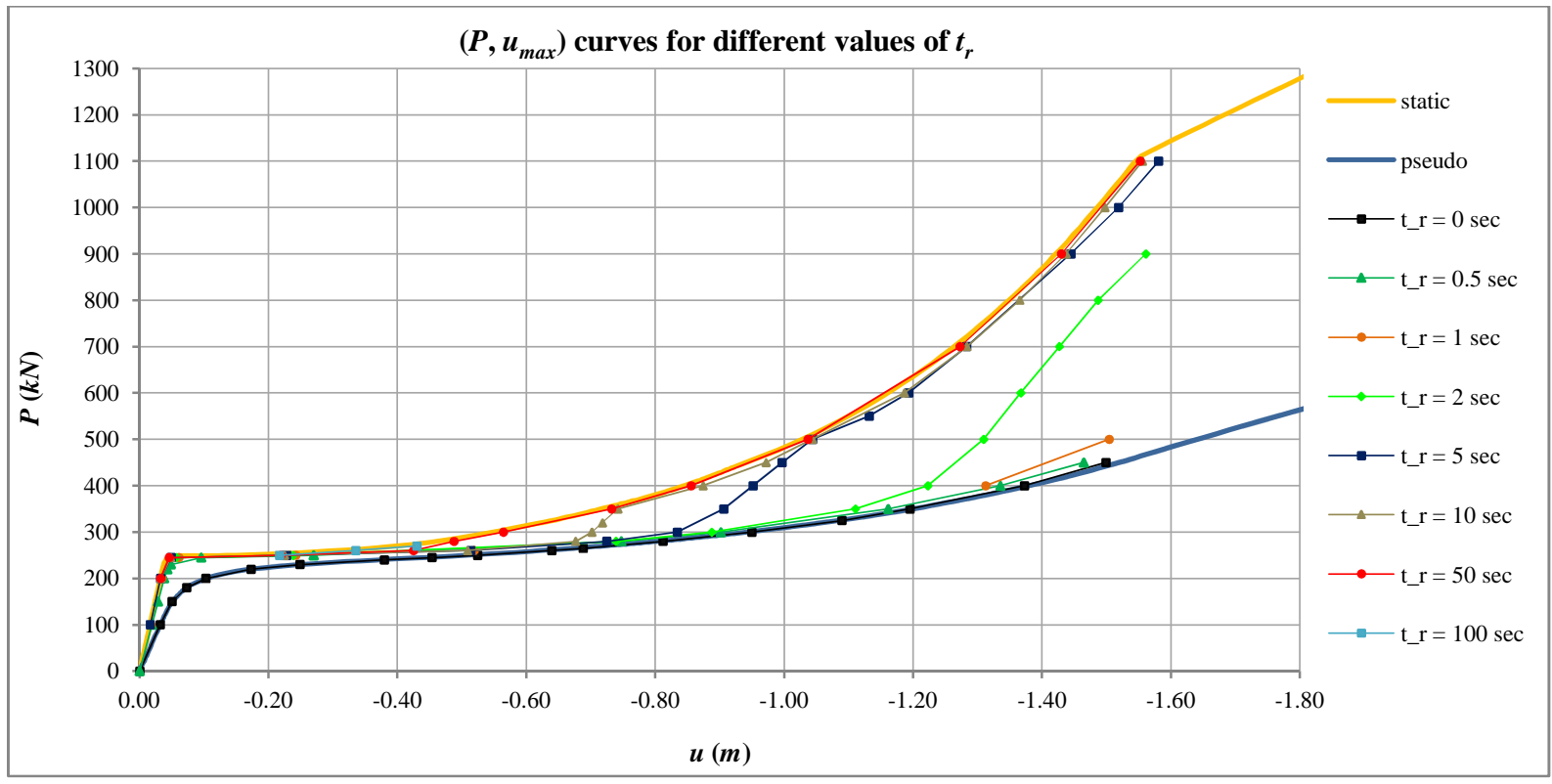

Fig. 6: Maximal dynamic displacement according to the value of the load and its rise time

This method is based on energetic considerations. When the maximum displacement is reached, the external work $W$ is equal to the internal strain energy $U$ that can be estimated as the area under the static curve until the value of the maximum displacement $u_{\max }$ :

$$
W=U \quad \Leftrightarrow \quad P \cdot u_{\max }=\int_{0}^{u_{\max }} P(u)_{s t a t} \cdot d u
$$

Thus, for any value of $u_{\max }$, the level of the instantaneous load $P$ causing this maximum displacement can be deduced from Eq. (1) and, eventually, the pseudo-static curve can be entirely derived from the static one. Numerical nonlinear dynamic analyses performed for different values of the instantaneous load $P$ confirmed the validity of this approach (see the black curve labelled " $t_{r}=0$ " in Fig. 6).

Obviously, the maximum displacement corresponding to a force $P$ will always be situated between the static displacement $\left(t_{r} \rightarrow \infty\right)$ and the displacement caused by the sudden application of the load $\left(t_{r}=0\right)$. As a consequence, every $\left(P, u_{\max }\right)$ curve will lie between the static and the pseudo-static ones all along, for any value of $t_{r}$. As a general rule, for a given value of $P$, the displacement $u_{\max }$ tends to decrease when $t_{r}$ increases (the dynamic effects decrease when the load is applied more slowly).

Different types of behaviour can already be highlighted from Fig. 6. For loads $P>P_{p l}$, two types of response are observed according to the loading parameters $P$ and $t_{r}$. For the first type, the maximum dynamic displacement $u_{\max }$ is greater than the static displacement $u_{\text {stat }}$ while, for the second one, $u_{\max }$ is very close to $u_{\text {stat }}$ (the dynamic curve $\left(P, u_{\max }\right)$ joins the 
static curve). Examples of both response types are presented in Fig. 7 and 8. For each of them, the dynamic curve, providing the time evolution of the displacement $u_{d y n}(t)$, is compared to the static curve $u_{\text {stat }}(t)$, representing the evolution of the displacement if the dynamic amplification is neglected. Accordingly, $u_{\text {stat }}\left(t^{*}\right)$ is the static displacement associated with the value of the applied load $P\left(t^{*}\right)$ at the time $t^{*}$.

A response of type 1 (Fig. 7) is obtained if the dynamic displacement reached after the formation of the beam plastic mechanism is larger than the static displacement corresponding to the final load $P$. Then, the system finally oscillates around a value of the displacement greater than this static displacement. In case of a behaviour of type 2 (Fig. 8), when the system yields and the displacement suddenly increases, the latter however remains smaller than the maximum static displacement corresponding to $P$. Afterwards, the dynamic curve $u_{d y n}(t)$ oscillates around a relatively constant value whilst the applied load continues to rise. Once the force $P(t)$ has increased enough so that the associated static displacement meets the dynamic displacement, the latter starts to increase again, oscillating around the static curve. Finally, the maximum dynamic displacement is close to $u_{\text {stat }}(P)$.

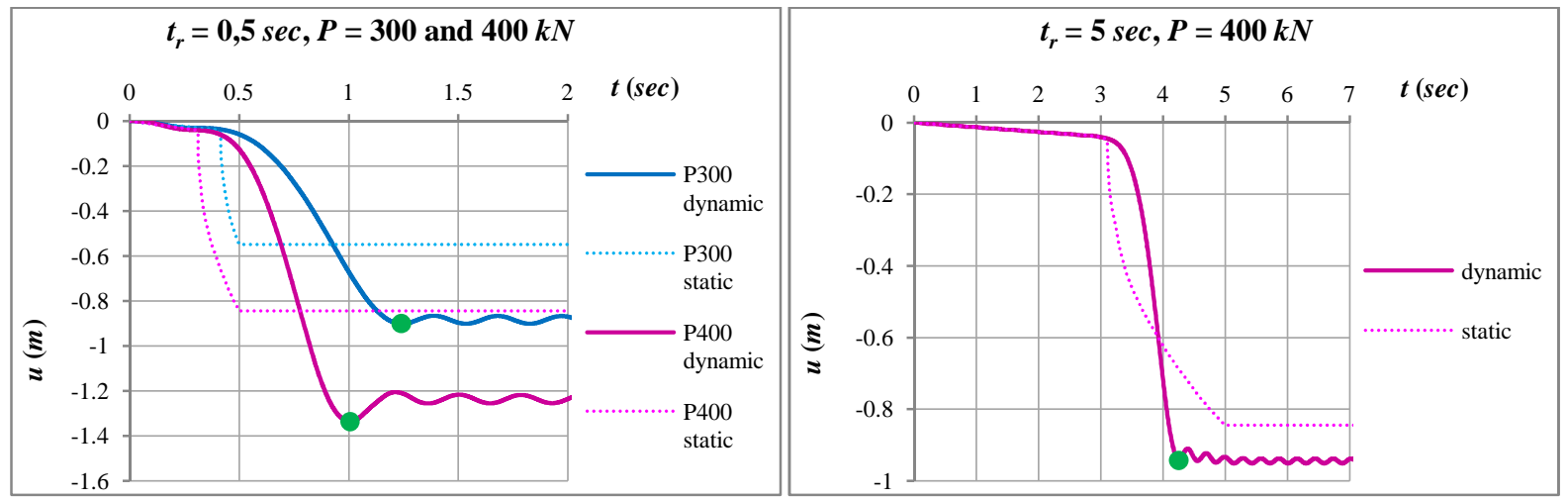

Fig. 7: Examples of the time evolution of the displacement for a type 1 response

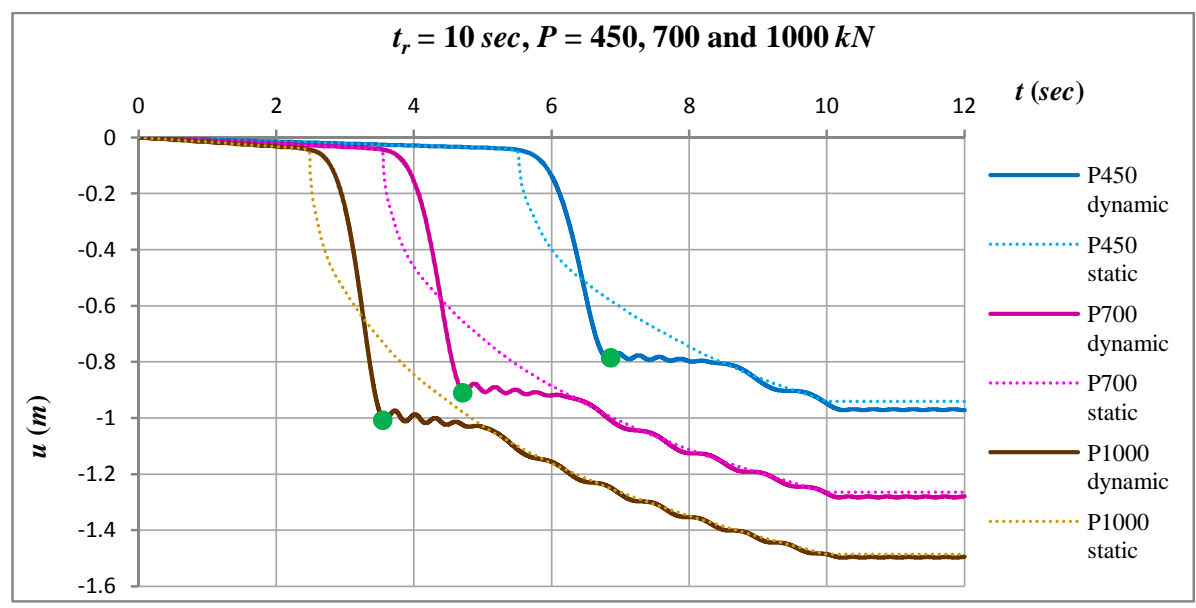

Fig. 8: Examples of the time evolution of the displacement for a type 2 response

The time evolution of the displacement for both response types can be explained as follows. When the plastic mechanism forms, the displacement rapidly increases and a distinct change in the slope of the curve $u_{d y n}(t)$ is observed. However, due to its inertia, the system starts moving progressively and, at the beginning, the displacement remains below the static displacement $u_{\text {stat }}(t)$ corresponding to the applied load $P(t)$. The system starts to accelerate and 
the dynamic displacement gets closer to the static one. Then it exceeds the latter and the velocity of the system begins to decrease. When the velocity is reduced to zero (which corresponds to the first maximum of the dynamic displacement curve, for a value of the displacement called $u_{\text {plateau }}$ - green point in Fig. 7 and Fig. 8), a "stabilisation" of the system is observed and the curve $u_{d y n}(t)$ is characterised by a sort of plateau. This may occur for a displacement $u_{\text {plateau }}$ smaller or greater than the static displacement associated with the final load; and that is what distinguishes the two behaviour types. In the first case (type 1 - Fig. 7), $u_{\text {plateau }}>u_{\text {stat }}(P)$ and the plateau is infinite. In the second case (type 2 - Fig. 8), $u_{\text {plateau }}<$ $u_{\text {stat }}(P)$ and the plateau is then limited by the static curve: it carries on until the applied force $P(t)$ has sufficiently increased so that the corresponding static displacement equals the dynamic displacement. Afterwards, the dynamic curve oscillates around the static one and finally stabilises at a level close to $u_{\text {stat }}(P)$.

As far as the internal forces are concerned, the axial load in the beams remains very small before the appearance of the three plastic hinges. When the moment in the middle and at the extremities of the double-beam reaches the plastic value $M_{p l}$, the mechanism forms and the displacement rapidly increases inducing significant membrane forces to develop in the beams. As a consequence, the moment acting in the plastic hinges decreases with respect to the plastic interaction between $M$ and $N$. At the end, oscillations of $M$ and $N$ are observed while the displacement is oscillating around a constant value. The amplitude of the oscillations of the tension force is limited since the amplitude of the variations of the displacement is also small. It is different for the moment that varies more importantly, in phase with the oscillations of $u$. To this phenomenon corresponds a succession of elastic unloadingsreloadings that can be observed using the $M-N$ interaction diagram or the $M-u$ and $N-u$ curves. Moreover, it is interesting to note that the maximum axial load in the beams, which is obtained when $u_{\max }$ is reached, is equal to the tension force that would have developed if this displacement had been statically reached. Accordingly, this membrane force can be deduced from the sole knowledge of the maximum dynamic displacement $u_{\max }$ and the static response of the substructure.

\subsection{Simplified approach to estimate the maximum dynamic displacement}

The objective of this section is to develop a simplified method to estimate the maximum displacement reached for a given loading condition $\left(P, t_{r} / T\right)$ with $P>P_{p l}$. Then, it would be possible to predict the required deformation capacity and tension resistance of the structural members.

In view of the shape of the $\left(P, u_{\max }\right)$ curves (Fig. 6), the concept is to approximate them, beyond the plastic plateau, using segmented curves established as follows (Ref. [9]):

- segment 1: horizontal line at the level of $P_{p l}$;

- segment 2: pseudo-static curve;

- segment 3: vertical line between the pseudo-static and the static curve, at the abscissa named $u_{\text {trans }}$ at which the actual $\left(P, u_{\max }\right)$ curve joins the static curve;

- segment 4: static curve.

An example of such a curve is presented in Fig. 9 (namely "correct" approximate curve in red). The aim of the procedure developed below is to predict such a curve approaching the "real" $\left(P, u_{\max }\right)$ curve obtained using several finite element analyses (performed with the software Finelg for $t_{r}=10 \mathrm{sec}$ in the example - purple curve in Fig. 9). For too low values of the $t_{r}$ to $T$ ratio, the dynamic curve does not join the static one and segments 3 and 4 cannot be 
defined (see Fig. 6). It is also possible that segment 2 does not exist (if the $\left(P, u_{\max }\right)$ curve joins the static curve before segment 1 has reached the pseudo-static curve).

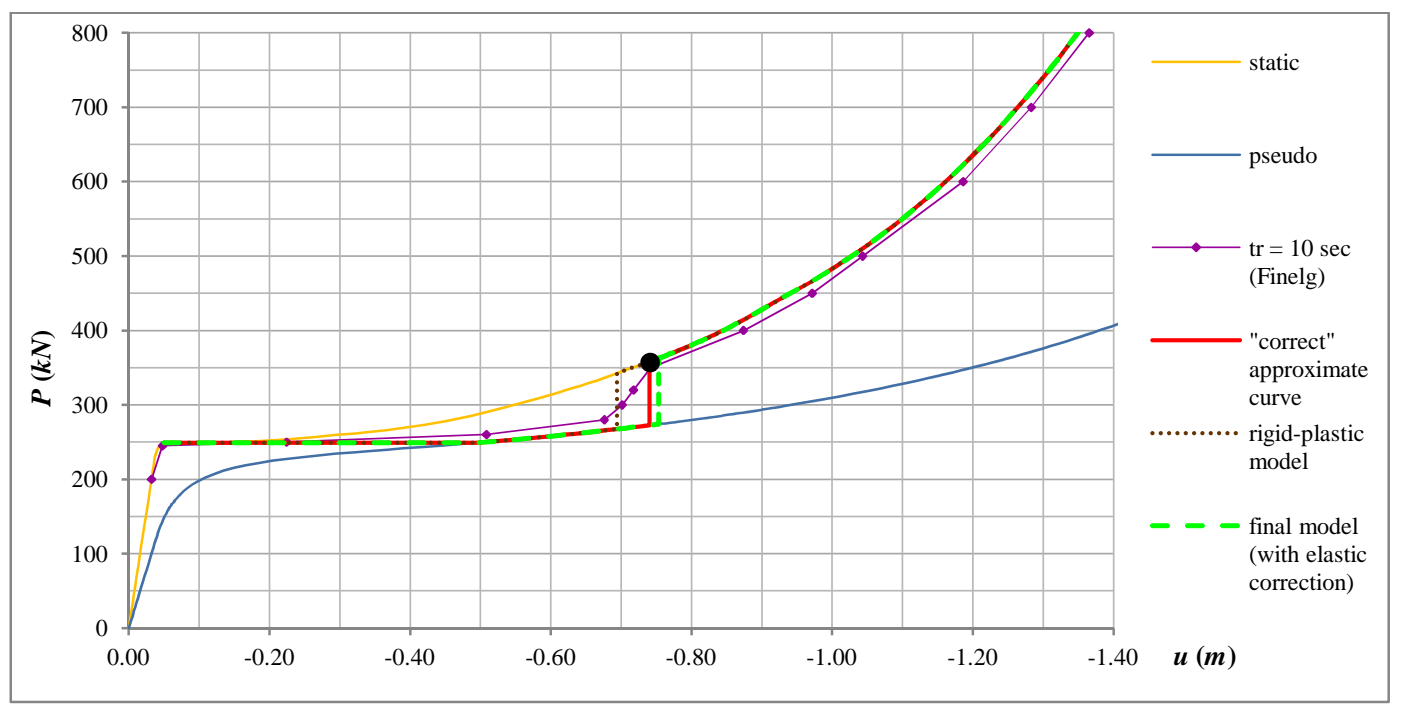

Fig. 9: Example of an approximate dynamic curve $\left(P, u_{\max }\right)_{a p p r}$

Once the static curve is known, the pseudo-static curve can directly be deduced and the only thing that is still to be determined to be able to draw the previously defined approximate curve is the value of $u_{\text {trans. }}$. The point $\left(P_{\text {trans }}, u_{\text {trans }}\right)$ at which the dynamic curve $\left(P, u_{\max }\right)$ associated with a given value of $t_{r} / T$ joins the static curve (black point in Fig. 9 ) corresponds to a transition between the two response types previously described. Indeed, we have $u_{\max }>$ $u_{\text {stat }}$ for $P<P_{\text {trans }}$ (type 1 ) and $u_{\max } \approx u_{\text {stat }}$ for $P>P_{\text {trans }}$ (type 2). As explained before, the behaviour type is governed by the value of the displacement $\left(u_{\text {plateau }}\right)$ when the velocity is reduced to zero for the first time after the formation of the plastic mechanism. In fact, type 1 corresponds to $u_{\text {plateau }}>u_{\text {stat }}(P)$ while type 2 is associated with $u_{\text {plateau }}<u_{\text {stat }}(P)$.

Consequently, if $u_{\text {plateau }}$ could be evaluated for a given loading, then the approximate dynamic curve $\left(P, u_{\max }\right)_{a p p r}$ corresponding to a fixed value of $t_{r}$ (or $\left.t_{r} / T\right)$ could be established following this procedure:

- evaluation of the displacement $u_{\text {plateau }}$ for different values of $P$ and comparison with the static displacement $u_{\text {stat }}(P)$;

- identification of the force for which $u_{\text {plateau }}$ equals $u_{\text {stat }}(P)$ : this value of the load is $P_{\text {trans }}$

- deduction of $u_{\text {trans }}=u_{\text {stat }}\left(P_{\text {trans }}\right)$ from the static curve;

- determination of the complete curve $\left(P, u_{\text {max }}\right)_{\text {appr }}$.

In order to carry out the first stage of this procedure, a simplified model is needed to estimate $u_{\text {plateau }}$. The development of this model is explained below.

The model is based on energetic considerations and consists in expressing that the work done by the external force is equal to the sum of the kinetic energy and the deformation energy (Eq. (2)). Considering a rigid-plastic behaviour for the beams (while the horizontal spring is defined by an elastic behaviour law), this deformation energy simply includes the work related to the plastic hinges rotation and the elastic energy included in the lateral spring.

$$
W_{e}=E_{\text {kinetic }}+E_{\text {defo }}=E_{\text {kinetic }}+E_{\text {hinges }}+E_{\text {spring }}
$$


This equation can be expressed as follows:

$$
\int P(u) \cdot d u=\frac{1}{2} \cdot M_{g} \cdot \dot{u}^{2}+4 \cdot \int M(\theta) \cdot d \theta+\int F_{K}\left(\delta_{K}\right) \cdot d \delta_{K}
$$

in which:

$M_{g}=1 / 3 \cdot m \cdot 2 \cdot l_{0}=1 / 3 \cdot M_{t o t}$ is the generalised mass of the system,

$\theta$ is the rotation of each plastic hinge and $M$ the corresponding plastic bending moment,

- $\delta_{K}$ is the elongation of the horizontal spring and $F_{K}$ the force it is submitted to.

Eq. (3) was further developed considering:

- the $M-N$ plastic interaction in the plastic hinges (the decrease of the plastic resistant moment of the profile cross-section is expressed through a function of the normal force):

$$
M_{p l}(N)=M_{p l} \cdot f(N(u))=M_{p l}(u) \quad<M_{p l}
$$

the axial elongation of the beams using:

$$
l=l(N)=l_{0}+2 \cdot \delta_{N} \approx l_{0}+2 \cdot \frac{N(u)}{K_{N}}=l(u) \quad>l_{0}
$$

where $\delta_{N}$ is the elongation of half a beam and $K_{N}$ the associated elongation stiffness (for more information about these parameters, see [7]).

moderate displacements meaning that (Fig. 10):

$$
\theta \approx \frac{u}{l}(\approx \sin \theta \approx \operatorname{tg} \theta) \text { and } \cos \theta \approx 1-\frac{\theta^{2}}{2} \approx 1-\frac{u^{2}}{2 \cdot l^{2}}
$$

The validity of this assumption is discussed at the end of this section.

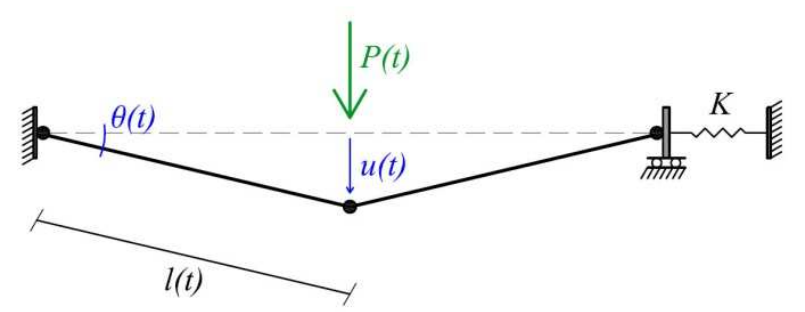

Fig. 10: Considered system and main definitions

Finally, the following movement equation could be derived from Eq. (3), using Eqs. (4), (5) and (6) and making a few conservative simplifications:

$$
M_{g} \cdot \ddot{u}(t)+\frac{4 \cdot M_{p l}}{l(u)} \cdot f(N(u))+\frac{2 \cdot K}{l(u)^{2}} \cdot u^{3}(t)=\frac{P}{t_{r}} \cdot t
$$

with: 


$$
\begin{aligned}
& \quad N(u)=\frac{K \cdot \delta_{K}}{\cos \theta} \approx \frac{K \cdot \frac{u^{2}}{l(u)}}{1-\frac{u^{2}}{2 \cdot l(u)^{2}}} \approx K \cdot \frac{u^{2}}{l(u)} \text { and } \quad N\left(u, l_{0}\right) \approx K \cdot \frac{u^{2}}{l_{0}}, \\
& l(u) \approx l_{0}+2 \cdot \frac{N\left(u, l_{0}\right)}{K_{N}} .
\end{aligned}
$$

As Eq. (7) has been developed considering the deformation energy related to the first loading of the system and neglecting possible elastic unloading, it is obviously only valid before unloading. In other words, the displacement curve $u(t)$ that can be obtained resolving this differential equation is only valid until its first maximum, which is $u_{\text {plateau }}$. Eq. (7) also assumes that $P(t)=P \cdot t / t_{r}$, which means it is only valid before the applied load has become constant (i.e. for $t<t_{r}$ - Fig. 5). However, these restrictions are of no consequence here. Indeed, what we are interested in is the determination of $u_{\text {plateau }}$ and what happens after is no concern. Moreover, as the final objective is the evaluation of $P_{\text {trans }}$, only responses relatively close to the intermediate situation between the two behaviour types are interesting; and, in such cases, the plateau always starts at a time $t_{\text {plateau }}<t_{r}$.

In order to solve Eq. (7), initial conditions have to be defined. In the considered rigidplastic system, the displacement and the velocity both equal zero until the plastic mechanism is formed. So the equation is resolved from the time $t_{p l}$, with the initial conditions (Fig. 11):

$$
\begin{aligned}
& u_{0}=u\left(t_{p l}\right)=0, \\
& \dot{u}_{0}=\dot{u}\left(t_{p l}\right)=0 .
\end{aligned}
$$

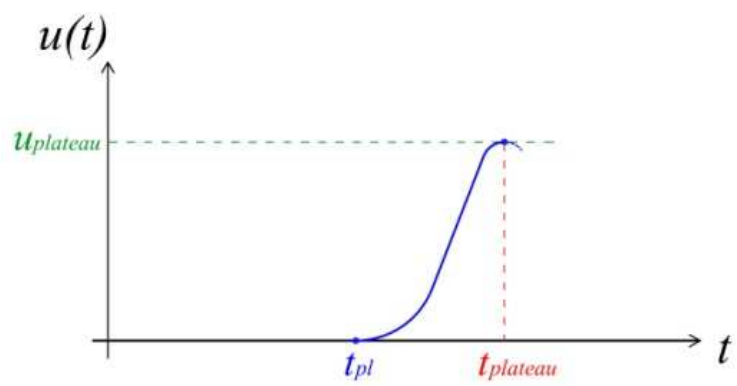

Fig. 11: Typical response of the system defined on the basis of the model

Unfortunately, this equation has no analytical solution and thus numerical calculation has to be performed to obtain the displacement curve $u(t)$, which is only valid until $u_{\text {plateau }}$ or $t_{r}$ is reached, as explained above. Using this model (Eq. (7)) for the prediction of $u_{\text {plateau }}$, the approximate curve labelled "rigid-plastic model" in Fig. 9 (brown dotted curve) could be established following the previously described procedure. It is observed that it still leads to underestimate the maximum dynamic displacement for values of the force $P$ close to $P_{\text {trans }}$.

The reason is that the model is based on the assumption of a rigid-plastic behaviour of the beams meaning that the elastic displacement reached before the formation of the plastic mechanism is neglected. If this displacement is added to the values of $u_{\text {plateau }}$ obtained using the proposed rigid-plastic model (Eq. (7)), a better estimation of the displacement $u_{\text {trans }}$ is found. Finally, the last curve depicted in Fig. 9 is obtained (curve named "final model with 
elastic correction"), which is very close to the "correct" approximate curve it was meant to approach.

For the considered example, it can be observed that the plastic hinges rotation does not exceed $7^{\circ}$ in the domain of deformation in which Eq. (7) is used and the moderate displacements hypothesis (Eq. (6)) is thus justified. Indeed, this movement equation is used to determine $u_{\text {plateau }}$ for increasing values of $P$ until $u_{\text {trans }}$ is identified, according to the procedure described at the beginning of this section to determine the approximate dynamic curve $(P$, $\left.u_{\max }\right)_{a p p r}$ for a given value of $t_{r}$. Consequently, the moderate displacements assumption (Eq. (6)) is valid as long as the rotation corresponding to the vertical displacement $u_{\text {trans }}$ remains smaller than about ten degrees. If it is not the case, large displacements should be taken into account and the relationships $\theta=\arcsin (u / l)$ and $\cos \theta=\sqrt{1-u^{2} / l^{2}}$ have to be considered instead of the previous simplifications of Eq. (6). The development of the movement equation in such conditions leads to more complex expressions and is not detailed herein.

\section{CONCLUSIONS AND PERSPECTIVES}

The investigations reported in the present paper are mainly dedicated to the study of the dynamic response of a simple substructure basically consisting of a double-beam subjected to a force $P$ at mid length and laterally restrained by a horizontal spring. Indeed, although not detailed herein, the dynamic behaviour of building frames further to the loss of a column was studied and demonstrated to show many similarities with the response of such a substructure in terms of displacement, yielding and internal forces.

The time evolution of the substructure displacement, yielding and internal forces was studied in details using finite element models. The influence of different properties of the structure (mainly its first period $T$ ) and of the loading conditions (the value of the load $P$ and the rapidity with which it is applied, characterised by the rise time $t_{r}$ ) was highlighted. On the basis of the conclusions drawn from these analyses, a simplified method was developed to quantify the maximum dynamic displacement $u_{\max }$ suffered by a substructure under a given loading condition $\left(P, t_{r} / T\right)$. The proposed model is based on the system static response and has been validated through comparison to results of finite element dynamic simulations.

The extension of the procedure presented herein for the prediction of the substructure dynamic response to global frames losing a column is currently under investigation. A general model is being developed and implemented into a software that will be available in a near future for easy practical use. This model will permit the determination of the frame dynamic response on the basis of its static behaviour and the way the bearing capacity of the failing column is supposed to decrease. 


\section{REFERENCES}

[1] British Standards Institution. BS 5950-1:2000. Structural use of steelwork in buildings Part 1: Code of practice for design - Rolled and welded sections. UK, 2001.

[2] US Department of Defence. Unified Facilities Criteria UFC 4-023-03. Design of buildings to resist progressive collapse. USA, January 2005.

[3] US General Services Administration. Progressive collapse analysis and design guidelines. USA, June 2003.

[4] Marchand K.A., Alfawakhiri F. Facts for steel buildings - Blast and progressive collapse. American Institute of Steel Construction, Inc. USA, April 2005.

[5] European Committee for Standardization. prEN 1991-1-7. Eurocode 1: Actions on structure - Part 1-7 : General actions - Accidental actions. Final project team draft (stage 34). July 2004.

[6] Ellingwood B.R., Dusenberry D.O. Building design for abnormal loads and progressive collapse. Computer-Aided Civil and Infrastructure Engineering 20, pp. 194-205. Blackwell Publishing, 2005.

[7] Demonceau J.-F. Steel and composite building frames: sway response under conventional loading and development of membrane effects in beams further to an exceptional action. $\mathrm{PhD}$ thesis. University of Liège, Belgium, 2008. (Freely downloadable at http://hdl.handle.net/2268/2740)

[8] Luu H.N.N. Structural response of steel and composite building frames further to an impact leading to the loss of a column. PhD thesis. University of Liège, Belgium, 2008.

[9] Comeliau L. Effets du comportement dynamique des structures de bâtiments en acier suite à la ruine accidentelle d'une colonne portante. Master thesis. University of Liège, Belgium, 2009. (Freely downloadable at http://hdl.handle.net/2268/32284)

[10] Comeliau L., Demonceau J.-F., Jaspart J.-P. Robustness of steel and composite buildings under impact loading. Proceedings of SDSS' Rio 2010 International Colloquium - Stability and Ductility of Steel Structures, Vol. 1, pp. 393-400. Rio de Janeiro, 2010.

[11] Comeliau L., Demonceau J.-F., Jaspart J.-P. Robustness of building frames further to a column loss - Substructure approach with account of dynamic effects. Proceedings of EUROSTEEL $2011-6^{\text {th }}$ European Conference on Steel and Composite Structures, Vol. C, pp. 2481-2486. Budapest, Hungary, 2011.

[12] FINELG user's manual. Nonlinear finite element analysis program. Version 9.0, 2003.

[13] Vlassis A.G. Progressive collapse assessment of tall buildings. Thesis submitted in fulfilment of the requirements for the degree of Doctor of Philosophy of the University of London and the Diploma of Imperial College London, UK, 2007. 
[14] Izzuddin B.A., Vlassis A.G., Elghazouli A.Y., Nethercot D.A. Progressive collapse of multi-storey buildings due to sudden column loss - Part I: Simplified assessment framework. Engineering Structures 2008:30(5):1308-18. 